\title{
EFFECTS OF NEUROMUSCULAR BLOCK REVERSAL WITH SUGAMMADEX VERSUS NEOSTIGMINE ON POSTOPERATIVE RESPIRATORY OUTCOMES AFTER MAJOR ABDOMINAL SURGERY. A RANDOMIZED CONTROLLED TRIAL.
}

Alday E., Muñoz M., Mata E., Alvarez C., Planas A. Hospital Universitario La Princesa, Madrid, Spain.

Background: Postoperative pulmonary complications may be reduced when neuromuscular block is reverted with sugammadex due to a lower incidence of residual block and to impairment of diaphragm function after neostigmine administration.

Objectives: The aim of the study is to compare lung function after major abdominal surgery when reversal of neuromuscular blockade is carried out with sugammadex or neostigmine.

Design: A randomized controlled trial.

Setting: A tertiary teaching hospital in Spain.

Patients: Adults scheduled for major abdominal surgery under combined general and epidural anaesthesia with no contraindications for any of the study drugs.

Interventions: Spirometry and lung ultrasound were performed before surgery and one hour and 24 hours postoperatively. Differences in mean changes from baseline were analyzed with repeated measures ANOVA.

Main outcomes measures: Forced vital capacity (FVC) loss, rate and size of atelectasis one hour after surgery in patients reverted with $40 \mu \mathrm{g} / \mathrm{kg}$ of neostigmine versus $4 \mathrm{mg} / \mathrm{kg}$ of sugammadex.

Results: One hundred and twenty-six patients were included for the main analysis.

\begin{tabular}{|c|c|c|}
\hline & Neostigmine $(n=64)$ & Sugammadex $(n=62)$ \\
\hline \multicolumn{3}{|l|}{ Surgery procedure n (\%) } \\
\hline Gastric/small bowel n (\%) & 7 (10.94) & $6(9.67)$ \\
\hline Liver resection n (\%) & $7(10.94)$ & $8(12.9)$ \\
\hline Pancreatic resection n (\%) & $6(9.38)$ & $4(6.45)$ \\
\hline Colorectal n (\%) & $41(64.06)$ & $39(62.9)$ \\
\hline Others n (\%) & $3(4.68)$ & $5(8.06)$ \\
\hline \multicolumn{3}{|l|}{ Surgical approach } \\
\hline Laparoscopic n (\%) & 39 (60.94) & 30 (48.39) \\
\hline Open surgery n (\%) & $25(39.06)$ & $32(51.61)$ \\
\hline \multicolumn{3}{|l|}{ Anesthetic management } \\
\hline tV ml/kg mean $\pm S D$ & $8.06 \pm 1.12$ & $8.09 \pm 1.19$ \\
\hline PEEP mean \pm SD & $7.68 \pm 5.6$ & $7.70 \pm 2.49$ \\
\hline $\mathrm{FiO}_{2}(\mathrm{mmHg})$ mean $\pm \mathrm{SD}$ & $0.51 \pm 0.7$ & $0.52 \pm .0 .9$ \\
\hline Rocuronium dose (mg) media \pm SD & $112.45 \pm 41.4$ & $125.56 \pm 39.06$ \\
\hline $\begin{array}{l}\text { Reversal drug dose }(\mu \mathrm{g} / \mathrm{kg} \text { or } \mathrm{mg} / \mathrm{kg}) \\
\text { mean } \pm \text { SD }\end{array}$ & $39.33 \pm 2.7$ & $3.96 \pm 0.43$ \\
\hline $\begin{array}{l}\text { Intraoperative balance }(\mathrm{ml} / \mathrm{kg} / \mathrm{h}) \text { mean } \\
\pm \text { SD }\end{array}$ & $5.93 \pm 4.24$ & $5.15 \pm 2.87$ \\
\hline Surgery duration $(\min )$ mean \pm SD & $216.95 \pm 97.20$ & $230.40 \pm 105.46$ \\
\hline $\mathrm{TOF}=0$ before reversal $\mathrm{n}(\%)$ & $3(4.76) *$ & $13(21.31) *$ \\
\hline Residual NMB (TOF<90\%) n (\%) & $45(71.73)$ ** & $19(31.15)$ ** \\
\hline Residual NMB (TOF<70\%) n (\%) & $11(17.46)$ & $4(6.53)$ \\
\hline
\end{tabular}

\begin{tabular}{lllll}
\hline & Neostigmine & & Sugammadex & \\
\hline & $1^{\text {a }}$ hour $(\mathrm{n}=64)$ & 24 hours $(\mathrm{n}=61)$ & $1^{\text {ahour }}(\mathrm{n}=62)$ & 24 hours $(\mathrm{n}=58)$ \\
VAS =0 $\mathbf{n}(\%)$ & $39(60.94)$ & $24(39.34)$ & $32(51.61)$ & $34(58.6)$ \\
VAS 1-4 $\mathbf{n}(\%)$ & $22(34.38)$ & $27(44.6)$ & $33(35.48)$ & $17(29.31)$ \\
VAS 5-7 $\mathbf{n}(\%)$ & $3(4.69)$ & $10(16.39)$ & $7(11.29)$ & $7(12.07)$ \\
VAS 8-10 $\mathbf{n}(\%)$ & $0(0)$ & $0(0)$ & $1(1.61)$ & $0(0)$ \\
VAS: Visual analogic scale. & & & \\
Postoperative pain & & &
\end{tabular}

\begin{tabular}{|c|c|c|c|}
\hline & $\begin{array}{l}\text { Basal FVC (I) } \\
\text { mean } \pm \mathrm{SD} \\
\left(\mathrm{Cl}_{95 \%}\right)\end{array}$ & $\begin{array}{l}1^{\text {st }} \text { hour FVC (I) } \\
\text { mean } \pm \text { SD } \\
\left(\mathrm{Cl}_{95 \%}\right)\end{array}$ & $\begin{array}{l}24^{\text {th }} \text { hours FVC } \\
\text { (I) mean } \pm \text { SD } \\
\left.\text { (Cl }\left.\right|_{95 \%}\right)\end{array}$ \\
\hline Neostigmine $n=64$ & $\begin{array}{c}2.98 \pm 0.13 \\
(2.76-3.24)\end{array}$ & $\begin{array}{c}2.5 \pm 0.12 \\
(2.27-2.74)\end{array}$ & $\begin{array}{c}2.23 \pm 0.11 \\
(2.01-2.46)\end{array}$ \\
\hline Sugammadex $n=62$ & $\begin{array}{c}3.02 \pm 0.13 \\
(2.76-3.29)\end{array}$ & $\begin{array}{c}2.58 \pm 0.12 \\
(2.35-2.81)\end{array}$ & $\begin{array}{c}2.22 \pm 0.11 \\
(1.99-2.44)\end{array}$ \\
\hline
\end{tabular}

* $p$-value $=0.007^{* *} p$-value $<0.001$

\begin{tabular}{lcccc}
\hline & \multicolumn{2}{c}{ Neostigmine } & \multicolumn{2}{c}{ Sugammadex } \\
\cline { 2 - 5 } & 1 10 hour & $24^{\text {th }}$ hours & 1 o hour & $24^{\text {th }}$ hours \\
Atelectasis $(\mathbf{n}, \%)$ & $24(39.34 \%)$ & $43(74.14 \%)$ & $18(29.51 \%)$ & $39(66.1 \%)$ \\
Size $\left(\mathrm{cm}^{2}\right)$ & 14.8 & 19.07 & 8.0 & 18.93 \\
& $\left(\mathrm{Cl}_{95 \%}: 6.58-22.03\right)$ & $\left(\mathrm{Cl}_{95 \%}: 13.98-24.16\right)$ & $\left(\mathrm{Cl}_{95 \%}: 5.78-10.22\right)$. & $\left(\mathrm{Cl}_{95 \%}: 13.55-24.31\right)$ \\
\hline
\end{tabular}

Postoperative lung ultrasounds

Conclusions: We could not find differences in pulmonary function after reversal with sugammadex or neostigmine in a high-risk population. 